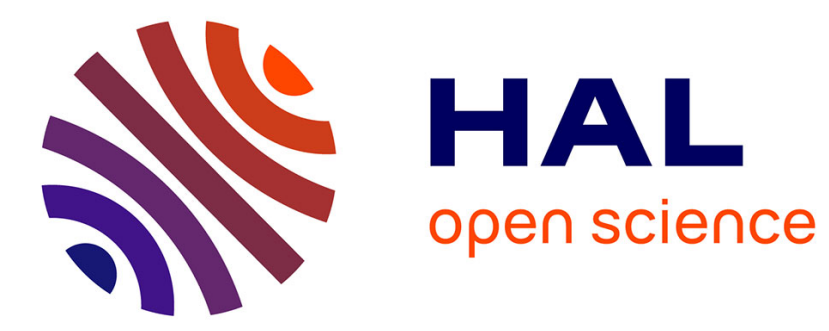

\title{
Les savoirs profanes et l'intelligence du web
}

Cécile Méadel

\section{To cite this version:}

Cécile Méadel. Les savoirs profanes et l'intelligence du web. Hermès, La Revue - Cognition, communication, politique, 2010, 57, pp.119-126. hal-00923094

\section{HAL Id: hal-00923094 \\ https://hal-mines-paristech.archives-ouvertes.fr/hal-00923094}

Submitted on 1 Jan 2014

HAL is a multi-disciplinary open access archive for the deposit and dissemination of scientific research documents, whether they are published or not. The documents may come from teaching and research institutions in France or abroad, or from public or private research centers.
L'archive ouverte pluridisciplinaire HAL, est destinée au dépôt et à la diffusion de documents scientifiques de niveau recherche, publiés ou non, émanant des établissements d'enseignement et de recherche français ou étrangers, des laboratoires publics ou privés. 


\section{Les savoirs profanes et l'intelligence du web}

\section{Cécile Méadel ${ }^{1}$}

La coupure entre savoir expert et savoir profane n'a pas attendu le développement des nouvelles technologies de l'information pour être mise en cause; le savoir expert n'est pas plus synonyme de vérité intangible que celui des profanes n'est tout entier du côté du subjectif et du local ; la vielle coupure entre les “ savants » (ceux qui ont acquis et parfois inventé de nouvelles connaissances) et les “ sachants (ceux dont la connaissance a été acquise dans l'action, par le savoir vécu transmis, mais aussi conquise en réponse à une question, à un problème personnel particulier) s'estompe vers un autre partage des savoirs (Delmas-Marty, 2007). Les travaux sur les controverses scientifiques ont montré comment les exigences de standardisation auxquelles doivent se soumettre les scientifiques aboutissent à une définition abstraite et générale mais aussi lacunaire et réduite du réel (Wynne, 1999); du coup, les savoirs profanes ne sont plus vus seulement comme l'expression de ceux qui ne savent pas ou ne comprennent pas, mais plutôt comme des connaissances et des points de vue qui peuvent être aussi établis, aussi fondés, aussi rationnels, aussi utiles que ceux des scientifiques (Epstein, 1995). Dès lors, comme toute connaissance, le savoir profane ne se réalise pas exclusivement dans des pratiques implicites ou informelles; il passe par des dispositifs, des façons de faire sens, des supports, des bases de données, des textes... et aussi, a minima, par la restitution de recherche d'informations, l'exploitation des données existantes, le recueil de documents, la diffusion de renseignements, de messages, de résultats.

On verra donc se déployer ces savoirs profanes, tout particulièrement dans certains champs d'intervention liés à la santé ou à l'environnement. Puis, on constatera qu'internet offre de nouvelles ressources qui ne se résument pas à la mise en ligne ou à la numérisation de contenus scientifiques mais qui offrent de nouvelles possibilités informatives et cognitives. Cela nous conduira à montrer que ces savoirs, qui s'appuient sur des ressources en accès libre, tirent leur efficacité de la participation de collectifs et de leur contrôle. Et pourtant, comme on le verra enfin, malgré l'ample travail de confrontation et de vérification accompli par ces groupes de "sachants", ces savoirs profanes accèdent difficilement au statut de connaissances stabilisées et reconnues.

\footnotetext{
${ }^{1}$ Centre de sociologie de l'innovation. Mines ParisTechn. cecile.meadel@ensmp.fr
} 


\section{De la mutation des ressources}

De ce point de vue, internet et surtout le web fournissent des ressources démultipliées. Ressources informatives d'abord puisqu'est mis à la disposition des internautes un énorme flux de textes, d'images et de sons, émanant d'acteurs de compétences et d'appartenances diversifiées. L'information scientifique y est surabondante et omniprésente ; certes, comme le montrent plusieurs des articles de ce dossier d'Hermès (Mounier, Farchy \& Froissart, Vinck...), elle est aussi diffuse, hérissée de barrières, rendue artificiellement rare, souvent confuse, mais sa surabondance est réelle, au point que l'on a pu s'interroger, avec l'optimisme coutumier que provoquent les nouvelles technologies de l'information, sur la réduction de la fracture cognitive qu'elle pourrait autoriser. Cette information savante est omniprésente non seulement sous la forme “canonique » de l'article scientifique ou de ses avatars, mais aussi dans des formats plus adaptés à un public non spécialiste. Ce que le web favorise en effet de manière privilégiée n'est pas tellement la forme vulgarisée des savoirs, mais des formats différents qui ne relèvent ni de la popularisation, ni de la rhétorique académique ; il permet en effet le développement d'autres formats qui rendent compte de la science en train de se faire, de la connaissance non aboutie, en donnant à voir les discussions sur les pratiques et les méthodes au même titre que les théories (discutées traditionnellement elles dans les articles scientifiques). Outre Wikipedia et autres ressources pédagogiques ou éducatives, on citera comme illustration les blogs de chercheurs qui se multiplient et dans lesquels leurs auteurs peuvent décrire, à destination d'un lectorat intéressé mais non professionnel, leurs travaux et expliciter leur démarche (Blanchard, 2010)².

Ressources productives aussi puisqu'un des aspects les plus frappants d'internet est de fournir la possibilité pour tout individu équipé d'un ordinateur et d'une liaison internet de produire des informations et de les publier. Bien sûr, l'espace où ces informations seront éditées, la rhétorique du document, la visibilité de l'auteur, ses garants... contribueront à définir la taille et la composition du public potentiellement touché (ou pas touché, si le document produit reste, comme c'est souvent le cas, perdu dans l'immensité du web invisible ${ }^{3}$.

Comment se déploient donc ces savoirs profanes en présence de l'internet et avec ses ressources? La question a été principalement étudiée dans deux domaines, l'environnement et la santé, non parce qu'ils font seuls l'objet de productions profanes, mais d'une part parce que l'information y était, avant le développement des technologies de l'information, difficile d'accès et faiblement disponible et d'autre part parce que les parties prenantes y jouent un rôle moteur.

\footnotetext{
${ }^{2}$ Voir par exemple les sites “Hypothèses " avec ses carnets de recherche en sciences humaines ou sociales, “le c@fé des sciences»ou encore "Prisme de tête ».

${ }^{3}$ M. Bergman (2001) estime que les moteurs de recherche n'indexent que 2 ou 3\% du web.
} 


\section{S'informer, c'est faire connaissance(s)}

Si la quête d'informations sur internet devient une procédure généralisée, elle reste le plus souvent une procédure personnelle et conjoncturelle. La fabrique des connaissances commence dès lors que la recherche et la collecte d'informations font l'objet de reprise et de diffusion. C'est par exemple le cas des malades qui, ayant fait un difficile parcours pour comprendre leur maladie et trouver des informations valides, estiment qu'ils peuvent faire économiser du temps et de l'énergie à des gens confrontés au même problème en publiant les résultats de leur recherche dans un site web (Ziebland, 2004, Méadel, 2007). Les choix effectués, l'organisation des documents, les questions posées sont une traduction de l'information. Traduction entre des langues d'abord, une part importante de l'information scientifique étant publiée en anglais; la traduction se faisant transposition lorsqu'elle est accompagnée par un texte introductif qui fait le lien entre la démonstration scientifique, l'expérience collective des patients, la situation locale confrontée à celle de l'article. Mais traduction entre deux mondes également: pour transposer et faire comprendre le langage des spécialistes, mais aussi pour le reconstruire en fonction des interrogations des profanes, pour l'articuler à leur propre expérience, pour l'adapter à leur situation particulière. Le degré d'autonomie par rapport au savoir savant est variable. Dans le domaine de la santé, ces informations reproduisent bien souvent le partage des compétences traditionnels entre les scientifiques détenteurs du savoir et les patients en demande d'informations et d'expertise. Dans celui de l'environnement, on est plutôt dans l'exercice du pouvoir de vigilance des citoyens (Rosanvallon, 2006) qui assurent une veille assidue et d'autant plus autonome qu'elle se veut contre-expertise.

Quels que soient le degré de retraduction par rapport aux approches savantes, ce travail de collecte et d'adaptation accompli par des profanes pour rendre compte de la production savante, ne peut être réduit ni à une popularisation du savoir savant, ni au savoir d'usage dont parle John Dewey; il y a bien production propre ; elle peut n'être que traduction et adaptation, mais peut aussi s'autonomiser et acquérir ses formats et sa rhétorique propres, dans un régime particulier d'ouverture et de circulation des contenus.

\section{L'engagement collectif dans le savoir}

Cette production des internautes peut en effet revêtir des formats très différents. Si on prend le domaine de la santé, les documents élaborés par les patients ou parents de patients vont du témoignage très personnel d'un nouvel opéré sur son intervention à des productions cognitives élaborées sous forme de bases de données, recueil et sommation d'observations, formulation d'hypothèses... Certains groupes mettent en place de véritables bases de données à caractère épidémiologique, d'autres construisent des outils de médication informatisés; beaucoup produisent des bases bibliographiques argumentées. Un seul exemple, à propos des maladies mal repérées et prises en charge comme les anomalies vasculaires (Méadel, Oziel, à paraître) : un groupe francophone recueille des témoignages et photos d'enfants atteints, permettant de favoriser à la fois le 
diagnostic, de repérer les bonnes ressources médicales et d'aider aux choix thérapeutiques souvent controversés dans ce domaine; le caractère ouvert du site et la vigilance sur les contenus par la modératrice mais aussi le collectif de patients qui l'entourent permettent un repérage très extensif de cas et une agrégation de contenus à large échelle (à la fois d'un point de vue géographique et clinique). Cette production n'intervient pas seulement pour aider et informer les patients; elle est aussi mobilisée par les spécialistes car elle constitue une base de données unique sur ces maladies, leur dépistage, leur prise en charge... Sous un certain nombre de conditions, on remarque que ce savoir profane construit de manière ouverte peut ainsi apporter une contribution originale et utile au débat public comme à la connaissance savante et qu'il peut contribuer à enrichir la connaissance ou la prise en charge des maladies par les professionnels eux-mêmes.

La force de telles connaissances dans l'espace public est souvent liée à l'engagement d'un collectif, qu'il soit structuré en association ou pas. Les savoirs produits peuvent être comme dans l'exemple des maladies vasculaires à l'origine de la constitution du collectif, mais ils peuvent aussi être un produit d'une mobilisation, d'une montée en généralité d'un groupe d'acteurs. Ainsi l'association de pêcheurs à la mouche, étudiée par Christelle Gramaglia (2008 \& 2009) a-t-elle progressivement modifié son rôle traditionnel de "porte-parole» des poissons, chargée de rendre compte devant les autorités publiques de la qualité de ses peuplements piscicoles; face à la pollution croissante des sites, elle est devenue la représentantes de la rivière dans son ensemble. Les membres ont changé de qualification et ont dû recueillir et mobiliser les connaissances qui leur ont permis de mener un véritable activisme juridique, nourri à la fois par leur intime connaissance de la rivière, de ses transformations et de ses habitants et par leur connaissances de procédures et des textes dans le domaine très particulier du droit de l'environnement. Leur travail se fait production propre lorsque, constatant les limites des jugements rendus par les tribunaux, ils s'allient avec des juristes pour “ inventer» de nouvelles approches du droit de l'environnement et de nouvelles pratiques judiciaires transmissibles aux associations désireuses de se lancer dans des actions en justice. Les connaissances recueillies peuvent aller jusqu'à remettre en cause les paradigmes scientifiques dominants, comme l'a montré Phil Brown (2006) à propos du cancer.

Le caractère collectif de cette production de savoirs et sa très large accessibilité sur les sites ou listes des membres assurent un contrôle qualité sur les productions de ces profanes. Ainsi, a-t-on pu montrer (Esquivel et al., 2006) que les erreurs médicales apparaissant dans une liste de discussion électronique entre malades sont corrigées avec une extrême rapidité, inégalée par aucun support d'information professionnel. Pourtant, cette production est difficilement reconnue par les professionnels et experts.

\section{Une production contestée et contestataire}

Elle est en effet attaquée ou récusée, en particulier dans les lieux de débats scientifiques sur internet, au motif qu'elle n’obéirait pas aux canons scientifiques. 
La bataille rappelle celle qui oppose au XVIII ${ }^{\mathrm{e}}$ siècle les tenants de la " science mondaine "désireuse d'un public curieux, éclairé et varié, de mise en scène, de démonstration explicite, et la "science sévère " qui ne s'autorise que de ses pairs (Chappey, 2006). L'état des connaissances s'est depuis largement transformé ; les normes scientifiques ne sont plus l'apanage des seuls professionnels de la science : il y a toujours parmi les lecteurs d'une information à caractère scientifique quelques lecteurs compétents qui la recouperont, la testeront, la valideront. Les “ savoirs professionnels diffus » (Sintomer, 2008) sont ainsi mobilisés par les acteurs au service d'une cause à laquelle ils peuvent apporter leurs compétences techniques, tel ce médecin marié à une femme atteinte par la maladie de Parkinson aidant une association de patients à construire un outil de suivi de la médication, ou cet ingénieur expertisant une analyse bactériologique pour l'association de son quartier... "Cette forme de savoir tend à croître avec le développement de l'instruction et d'une " société de la connaissance », et sa mobilisation va d'ailleurs de pair avec le rôle important que jouent les couches moyennes intellectuelles portées par la modernité dans les nouveaux mouvements sociaux. » (ibid..).

Production récusée par son articulation avec le politique. On constate que, dans un certain nombre de circonstance, cette production profane contribue à nourrir le débat public autour de la santé ou de l'environnement et à alimenter la connaissance, ne serait-ce que celle des perceptions et réactions des citoyens et des patients. Cela a été par exemple le cas dans le débat anglais autour du vaccin ROR (rougeole, oreillons et rubéole) : le travail de recueil d'informations mené par les parents a pu leur permettre de construire une position cohérente et efficace face aux politiques de santé qui prônaient la vaccination de tous les enfants (Hardey, 2004). Il faut noter que le savoir profane change partiellement de statut de par cette conception collective. Les pouvoirs publics reconnaissent la validité des savoirs de la société civile; et les connaissances de cette dernière y acquièrent depuis longtemps, Prévil) un statut juridique dans la mesure où l'opinion des profanes est requise dans un certain nombre de procédures, comme par exemple les enquêtes publiques instituées pour les questions environnementales (Monnoyer-Smith, 2007).

Production attaquée enfin parce qu'elle serait motivée par des préoccupations personnelles, des motivations politiques qui empêcheraient ses auteurs d'analyser un phénomène en toute liberté et indépendance. Ainsi Chantal Aspe (2009) oppose-t-elle la connaissance-processus et la connaissance-produit. Le partage est difficile à ternir. La recherche de connaissances, y compris en science, est articulée à des objectifs qui lui sont extérieurs : les scientifiques ne l'emportent, comme Pasteur contre Pouchet (Farley \& Geison, 1991) ou Edison contre Charles Cros, que quand ils arrivent à intéresser et à mobiliser une large pluralité d'acteurs qui ont des intérêts tout autres que scientifiques à les soutenir. Par ailleurs, ne pas rechercher une connaissance pour elle-même, pour des savoirs formels, mais pour résoudre un certain nombre de problèmes, pour aider à affronter une situation ou une condition difficile permet de poser des questions qui renouvellent la connaissance en amenant vers des territoires inexplorés. Une telle posture oblige à 
examiner des nouvelles interrogations, ̀̀ prendre en compte de nouvelles hypothèses. On en revient à la notion de " passion contrariée » mobilisée par C. Gramaglia pour expliquer l'intense activité productive de ces pêcheurs à la mouche.

Ainsi, pour les listes de discussion que nous avons étudiées avec Madeleine Akrich (2004 et 2007), nous avons pu montrer qu'elles ont bien une activité collective qui le plus souvent ne débouche pas sur des formats traditionnels de mobilisation, à la manière des associations ou des syndicats, mais qui ne se limitent pas non plus à des effets purement individuels. En fait, les échanges sur ces listes, s’ils ne se convertissent pas en une action collective immédiate, semblent être largement repris au niveau individuel dans des formes de participation à des collectifs externes. Les listes apparaissent comme des sortes de laboratoires dans lesquels circulent et sont débattues des informations, se forgent des convictions, des identités, s'élaborent des options stratégiques qui pourront être reprises dans d'autres espaces publics.

Beaucoup de chercheurs se sont interrogés sur les raisons qui poussent les internautes ou du moins une minorité d'entre eux à ne pas seulement consommer de l'information, mais aussi à en produire, le plus souvent en l'absence de toute contrainte sociale, économique ou institutionnelle (Beuscart, 2009). En matière d'environnement comme de santé, on constate que, outre les motifs traditionnels mobilisés pour justifier cette activité : satisfactions personnelles, symboliques ou professionnelles, reconnaissance pour la compétence déployée, etc., cette production peut être motivée par un engagement dans une cause, l'engagement dans un combat, qu'il soit préalable au travail sur l'information (Granjon, 2002) ou qu'il en découle.

Le rôle des technologies de l'information, et tout particulièrement de l'internet, dans les mobilisations a été très largement étudié (F. Greffet et S. Wojcik, 2008 ; Granjon, 2002). Les travaux ont montré que ses caractéristiques propres (comme les formats de discussion et de participation, l'asynchronie des débats, les procédures d'archivage et de consultation, la caractérisation des participants, etc) influencent et modifient les modes d'argumentation et de mobilisation. Pourtant, ces approches importantes et fructueuses n'ont que peu analysé la production informative de ces groupes militants, ne les considérant que rarement comme des sources de connaissances diffusables et mobilisables en dehors de l'action circonscrite qui a suscité leur production. Or, comme nous avons pu le voir à propos des patients (Akrich et Méadel, 2002), ces collectifs peuvent aussi produire des savoirs non exclusivement réductibles à des situations locales et idiosyncratiques.

Des travaux commencent à être menés sur ces savoirs profanes, mais ils ignorent le plus souvent les médiations par lesquelles ils se constituent. Internet est considéré principalement comme un outil de coordination et de diffusion parmi d'autres sans que l'on s'interroge sur la manière dont le web, l'ouverture des bases de données, les procédures particulières qui permettent de collecter des informations dans le monde entier, de confronter différentes versions d'un même document, de discuter un texte, de le rédiger collectivement... contribuent à la fabrication de ces savoirs et 
à leur formatage. Ce qui est désormais étudié pour les savoirs scientifiques, devrait l'être pour les savoirs profanes!

\section{Biblio}

Madeleine Akrich, et Cécile Méadel, 2004, "Prendre ses médicaments / prendre la parole : les usages des médicaments par les patients dans les listes de discussion électroniques", Sciences sociales et Santé, 20, 1, pp 89-116. \& 2007, “De l'interaction à l'engagement : les collectifs électroniques, nouveaux militants dans le champ de la santé ", Hermès, 47

Chantal Aspe, 2009, "Diffusion des savoirs sur internet et interactions citoyennes : de la "connaissance-produit" à la "connaissance-processus" ", VertigO, La revue en sciences de l'environnement, hors-série 6 , décembre

Michael K. Bergman, 2001, "White Paper: The Deep Web: Surfacing Hidden Value ", The Journal of Electronic Publishing,

Jean-Samuel Beuscart, Eric Dagiral et Sylvain Parisie. 2009, "Sociologie des activités en ligne ", Terrains \& travaux, 1, 15, 3-28.

Antoine Blanchard, 2010, "Ce que le blog apporte à la recherche », in Marin Dacos (dir.), Read/Write Book, Marseille, Cléo, Coll. Édition électronique, 157-166.

Phil Brown et al., 2006, "A Lab of Our Own": Environmental Causation of Breast Cancer and Challenges to theDominant Epidemiological Paradigm ", Science Technology Human Values, 31, 499-536.

Jean-Luc Chappey, “Enjeux sociaux et politiques de la "vulgarisation scientifique ” en Révolution (1780-1810)", in Annales historiques de la Révolution française, Numéro 338, [En ligne], mis en ligne le : 20 février 2006. URL : http://ahrf.revues.org/document1578.html.

Mireille Delmas-Marty, 2007, “La démocratisation des savoirs », Rue Descartes, 55, février, avec Françoise Massit-Folléa.

Steven Epstein, 1995, "The Construction of Lay Expertise: AIDS Activism and the Forging of Credibility in the Reform of Clinical Trials ", Science, Technology \& Human Values, 20, 4.

Adol Esquivel, Funda Meric-Bernstam et Elmer Bernstam, “ Accuracy and self correction of information received from an internet breast cancer list: content analysis ", BMJ, 2006, 332, 7547, 939-942 http://dx.doi.org/10.1136/bmj.38753.524201.7C.

John Farley et Gerald Geison "Le débat entre Pasteur et Pouchet : science, politique et génération spontanée au XIXe siècle " in Callon et Latour (sous la dir.) (1991) La Science telle qu'elle se fait. Anthologie de la sociologie des sciences de langue anglaise. Éditions La découverte, Paris.

Christelle Gramaglia, 2008, “Des poissons aux masses d'eau : les usages militants du droit pour faire entendre la parole d'êtres qui ne parlent pas ", Politix, 21, 83. \& 2009, “ Passions et savoirs contrariés comme préalables à la constitution d'une cause environnementale », Revue d'anthropologie des connaissances, 3,3 .

Fabien Granjon, 2002. "Les militants internautes, passeurs, filtreurs et interprètes », Communication, 1, 11-32. 
Fabienne Greffet et Stéphanie Wojcik, 2008, “Parler politique en ligne. Une revue des travaux français et anglo-saxons ", Réseaux, 4,150.

Michael Hardey, 2004. “Internet et société: reconfigurations du patient et de la médecine? ", Sciences sociales et Santé, 22, 1, 21-44.

Cécile Méadel, 2006. “ Le spectre “ psy » ordonné par des parents d'enfants autiste. L'étude d'un cercle de discussion électronique », Politix, 73.

Cécile Méadel, David Oziel, à paraître, “L'expérience des patients, innovation dans la production de l'information médicale ", Orfagen.

Laurence Monnoyer-Smith, 2007, “Instituer le débat public: un apprentissage à la française », Hermès, 47

Carlo Prévil, 2009, “ Participation du public dans la gouvernance de l'environnement et du territoire : pour améliorer l'instrumentation ", VertigO - la resue électronique en sciences de l'ensironnement, Volume 9 Numéro $1 \mid$ mai, [En ligne], mis en ligne le 27 mai 2009. URL : http://vertigo.revues.org/8580. Consulté le 03 avril 2010.

Pierre Rosanvallon, 2006. La Contre-Démocratie. La politique à l'âge de la défiance. Paris, Seuil, 345 .

Yves Sintomer, 2008, “ Du savoir d'usage au métier de citoyen? », Raisons politiques 3, 31, 115-133.

Brian Wynne, 1999, “Une approche réflexive du partage entre savoir expert et savoir profane ", Les Cahiers de la sécurité intérieure, 38, 219-236.

Sue Ziebland, 2004, “ The importance of being expert: the quest for cancer information on the Internet », Social Science \& Medicine, 59, 9, pp.1783-1793. 